\title{
Thermodynamics of Molecular Recognitions between Antineoplastic Drug Taxol and Phosphatidylcholine
}

\author{
Erhan Süleymanoglu* \\ Gazi University; Faculty of Pharmacy; Department of Pharmaceutical Chemistry; Hipodrom; 06330-Ankara - \\ Turkey
}

\begin{abstract}
The aim of this study was to study the basic features of Taxol recognition with phospholipids by applying the thermodynamic and spectroscopic measurements. The obtained information could be used further for deductions on its precise cellular and pharmacological mechanisms of action, on improvements of its solubility properties by phospholipids, as well as for designing the novel lipidic carriers for drug delivery.
\end{abstract}

Key words: Antineoplastic agents, chemotherapy, Taxol, phoshatidylcholine, Taxol-membrane interactions, membrane fluidity

\section{INTRODUCTION}

Over the past two decades, among the several new chemotherapeutic agents, the taxanes have played a significant role in the treatment of various malignancies (Singla et al. 2002; Stohs, 2005; Hennenfent and Govindan, 2006; Safavy, 2008). These are a group of hydrophobic antineoplastic drugs, approved by Food and Drug Administration (FDA) to cure breast, ovarian, non-small-cell lung and prostate cancers (Stohs, 2005). As a member of this group, Taxol was discovered in 1960s within the frame of a National Cancer Institute study for screening the natural compounds with anticancer properties (Wall and Wani, 1995a; 1995b). It was identified as the crude extract from the bark of the North American pacific yew tree Taxus brevifolia, subsequently found to be effective against various tumors (Stohs, 2005). Unfortunately, its clinical testing was unsatisfactorily delayed due to limited sources of the pacific yew tree bark and its poor solubility
(Harlan, Jr., 2001; Wall and Wani, 1995a,b; Stohs, 2005). Therefore, the tedious procedure of the total synthesis of Taxol has always been a challenging target of a number of research groups as an improved source of this promising anticancer drug (Zefirova et al. 2005).

Taxol (Paclitaxel) is a complex natural alkaloid diterpene compound with a molecular weight of 853 (amu) and is comprised of tetracyclic core (baccatin III) and ester-linked side chain attached to the 13th carbon of the latter. Pharmacological targets of the drug are cellular mirotubules with binding sites engaged, which biological functions are not yet clarified. Application of Taxol in cancer chemotherapy has been limited by its low aqueous solubility. Since this is regarded as an important issue for drug formulation, various solubilizing systems have been explored to improve its poor solubility. Among these, several approaches such as using co-solvents or emulsions, designing its new prodrug or analog forms as well as developing liposomal and

\footnotetext{
* Author for correspondence: esuleymanoglu@gazi.edu.tr
} 
micellar preparations of Taxol have been extensively tried (Nuijen et al. 2001; Singla et al. 2002; Safavy, 2008). However, none of these approaches has been satisfactory enough to be introduced to the clinical practice, mostly because of the lack of biocompatibility to meet the requirements of intravenous preparations. Of these, the use of lipidic carriers in the form of emulsions (Nornoo, 2008) nanoscale lipocores and liposomes (Terwogt, et al., 1997; Nuijen, et al. 2001; Singla et al. 2002; Castor, 2005; Safavy, 2008) for increasing the drug solubility are one of the most promising, but still awaiting further characterization.

In this respect, the objective of this study was to follow further the molecular recognitions between Taxol and phosphatidylcholine-one of the major components of eukaryotic cell membrane phospholipids. The goal to be achieved is twofold-both cell biological and biotechnological aspects are aimed. By further clarifying the precise mechanisms of Taxol-lipid interactions, better understanding of its pharmacological activities can be obtained, as well as designing the novel lipid based drug formulations with improved bioavailability properties would be possible. Since most of the commercial preparations of Taxol for intravenous infusion contain ethoxylated castor oil (Cremophor EL), it is interesting to study the pharmaceutical and physicochemical behaviour of the drug in similar solubilizing environments. Thus, well tolerated lecithin was chosen as a model emulsifying agent to simulate the parenteral formulation. The employed egg lecithin could be a good approach to currently used micellar solutions of egg lecithin and cholic acids as carrers for fatsoluble vitamin $\mathrm{E}$ (Cernevit) and vitamin $\mathrm{K}$ (Konakion MM) (Sznitowska et al. 2008). Having appreciated the importance of various physical forms of Taxol, possessing different therapeutic potentials, thermal analysis best fits for studying its solid states. More specifically, information on the physical states of drugs inside micelles or liposomes is useful in predicting formulation properties such as drug-release biodynamics and kinetics. Such loading into micelles or liposomal entrappment often leads to drug crystalization, whereas the drug molecules exist in molecularly disperced state at low loading. To better understand these phase transitions, a thermodynamic approach to follow Taxol-lipid recognitions is presented herein.

\section{MATERIALS AND METHODS}

\section{Materials}

Egg phosphatidylcholine was purchased from Avanti Polar Lipids (Alabaster, AL, USA). FTIRgrade $\mathrm{KBr}$ (22.186) and Paclitaxel were a product of Sigma Chem. Co., MI (USA). All the chemicals used were from the highest analytical grade.

\section{Methods \\ Differential Scanning Calorimetry}

Thermal analyses were carried out using PerkinElmer Pyris differential scanning calorimetry (DSC) instrument. Previously weighed and dried Taxol-lipid mixtures in desired ratios were placed in a hermetically sealed aluminium pans. Both the lipid and paclitaxel were dissolved in chloroform forming the final ratios and placed in aluminium pans prior to the measurements. The lipid concentration used throughout the measurements was $20 \mathrm{mg} / \mathrm{ml}$. As a control, a pan containing the dried paclitaxel of the same amount was prepared. Pacitaxel was disolved also in chloroform for comparative purposes. The cell was purged with Argone and all the measurements were made with a scan rate of $10^{\circ} \mathrm{C} / \mathrm{min}$.

\section{FTIR Spectroscopy}

Infrared spectra were recorded on IFS 66/S FTIR spectrometer (Bruker Analytische Messtechnik $\mathrm{GmbH}$, Karlsruhe, Germany), equipped with $\mathrm{He}-$ $\mathrm{Ne}$ laser detector and $\mathrm{KBr}$ beam splitter. $\mathrm{KBr}$ pellet method was employed as FTIR sampling technique. The samples were prepared by mixing the lipid and drug in the desired ratios and pressing the mixtures with a die press. Spectra were collected after short incubation of lipid with Taxol. Interferograms were accumulated over the spectral range $4000 \mathrm{~cm}^{-1}$ to $400 \mathrm{~cm}^{-1}$, with a nominal resolution of $2 \mathrm{~cm}^{-1}$ and a minimum of 320 scans. The criterion for the elimination of water effect from the spectra was based on the straight baseline between $1750 \mathrm{~cm}^{-1}$ and $2200 \mathrm{~cm}^{-1}$, where the water combination mode was located. As a control, the spectra of unbound Taxol was taken and compared with those reported in the literature previously. Afterwards, the spectra of egg phosphatidylcholine was recorded as the following. After the normalization of absorbance and baseline correction, the peak frequencies of the symmetric $\mathrm{CH}_{2}$ stretching, the asymmetric $\mathrm{P}=\mathrm{O}$ stretching and the asymmetric $\mathrm{N}-\left(\mathrm{CH}_{3}\right)_{3}$ stretcing peaks 
were determined by using the original software provided by the manufacturer. FTIR spectra were taken for each single component engaged in DNAlipid complex formation for all the combinations of binary mixtures Taxol-phosphatidylcholine in various ratios for comparison.

\section{RESULTS AND DISCUSSION}

The aim of this study was to investigate how Taxol recognized and bound to phospholipids and how it affected the packing and fluidity of membranes. The thermograms of DSC measurements could distinguish between the phospholipid gel and liquid crystalline phases. As the calorimetric data from the heating curves reflected essenially the same changes as those from the cooling curves, only endothermic curves were used to chracterize the thermotropic phase transitions of the binary drug-lipid mixtures. It was very interesing to study the excess drug effects by varying the drug concentration and keeping the lipid concentration fixed. This would give further insights into the concentration dependent Taxol effects, as well as into possible mechanisms of membrane deformation.

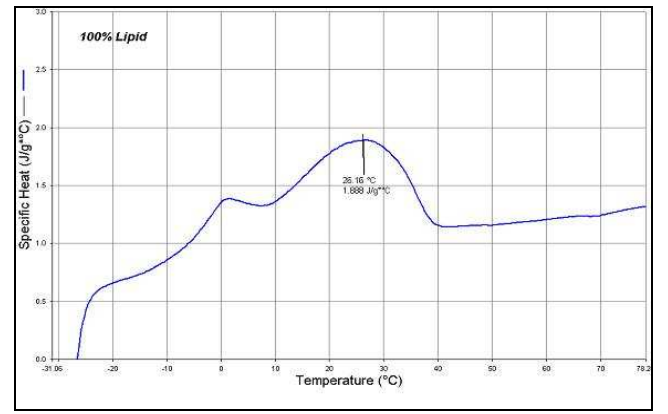

(a) A melting curve of a pure unbound phosphatidylcholine.

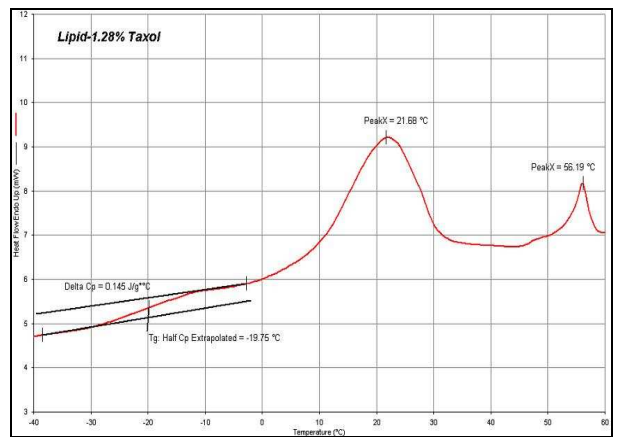

(b) A DSC thermogram depicting the melting profile of 1,28\% drug/lipid mixture.

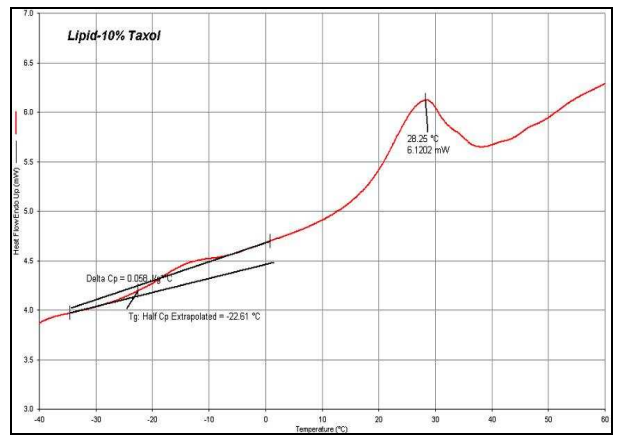

(c) A thermal melting profile of $10 \%$ drug/lipid mixture. 


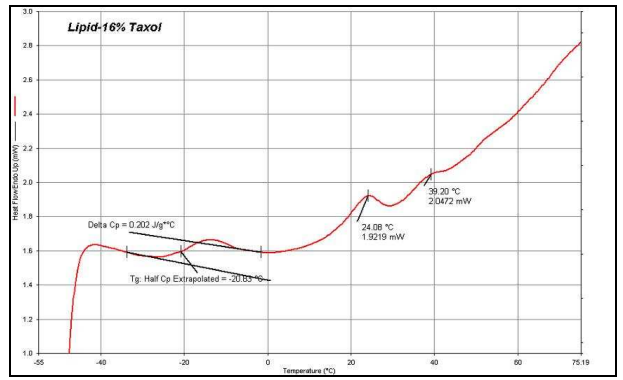

(d) A DSC thermogram showing the phase behaviour of $16 \%$ drug/lipid mixture.

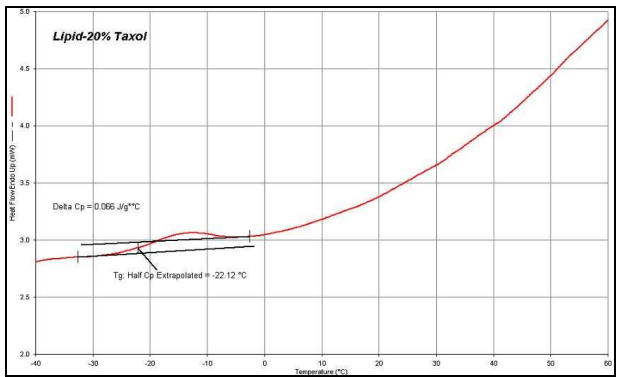

(e) A phase behaviour of $20 \%$ drug/lipid mixture.

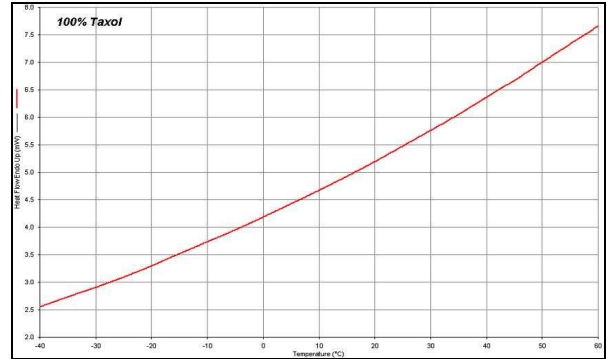

(f) A DSC scan of a pure unbound Taxol.

Figure 1 - DSC thermograms of unbound Taxol and its various mixtures with egg phosphatidylcholine in the desired ratios, as depicted. The thermodynamic parameters are assignet authomatically by the Pyris software of the DSC instrument. The samples were prepared as mentioned in the Materials and Methods.

Fig. 1 shows the endothermic phase transitions for binary mixtures of egg phosphatidylcholine and Taxol. Information on the physical state of drugs entrapped in micelles or in liposomes is very useful in predicting and designing improved formulation properties such as drug release kinetics from vesicles. The high loading of a drug with poor aqueous solubility, such as Taxol into vesicles usually leads to its crystallization, whereas the drug molecules exist in molecularly dispersed form at low loading profiles. Thermal analysis best fits for examining the crystallization features in simulated lipid environments. The first thermogram in Fig. 1 shows the heating behaviour of phosphatidylcholine alone, followed by several
DSC scans of Taxol-lipid complexes in various ratios in the solid state. All the thermograms differed from the heating characteristics of Taxol alone in the last thermogram, which showed no transitions at all, since it melted at much higher degrees at $216^{\circ} \mathrm{C}$. This suggested that the drug was homogeneously and molecularly dispersed within the surrounding lipid. Obviously, this recognion with lipid moleculs affected the crystalline structure of Taxol in a hermetically sealed aluminium pans.

In general, the addition of the drug induces certain perturbations or more specifically leads to microdomain formation of phospholipid molecules. When added even at low concentration 
to phosphatidylcholine, it gives rise to shift of the glass transition temperature $\left(\mathrm{T}_{\mathrm{g}}\right)$. The apperance of a second peak at $56.19^{\circ} \mathrm{C}$ indicated the formation of another phase. Obviously, drug-induced microdomain formation occured. Since such a phase transition was highly sensitive to existance of foreign molecule bound to polar parts of phospholipids, the changes in $\mathrm{T}_{\mathrm{g}}$ could not be explained only by specific molecular transitions. In $10 \%$ concentration, Taxol shifted the main phase transition temperature $\left(\mathrm{T}_{\mathrm{m}}\right)$ from 26.16 to $28.25^{\circ} \mathrm{C}$ (Fig. 1). However, the surrounding thermogram remained symmetric. On the other hand, the $T_{m}$ peak narrowed and its height diminisheed. When used at its lowest concentration, the drug shifted $\mathrm{T}_{\mathrm{m}}$ to lower degrees from 26.16 to $21.68^{\circ} \mathrm{C}$. However, the surrounding thermogram peak remained symmetric, while its width was narrowing. This effect increased the $T_{m}$ cooperativity of the phase transition and restricted the flexibility of phospholipids. In such a case, the highly ordered all-trans hydrocarbon phospholipid chain conformation existed. Gauche-(kinked) conformation, which leads to higher rotational freedom did not exist. The addition of $10 \%$ Taxol shifted the main phase transition temperature, giving rise also to appearance of a smaller second peak. This indicated that in higher amounts, the drug increased its affinity of binding to the phospholipids. In comparison with $\mathrm{T}_{\mathrm{m}}$, sharper changes were seen in glass transition temperature
$\left(\mathrm{T}_{\mathrm{g}}\right)$. Thus, in the presence of lower amounts of Taxol, the latter shifted from -19.75 to $-22.61^{\circ} \mathrm{C}$, while extending the peak width. It was interesting to note, that the same effect was observed when 10,16 and $20 \%$ concentrations of the drug were used (Fig. 1). These effects showed that when used in $1-10 \%$ concentraions, the drug increased the phospholipid fluidity by lowering $T_{m}$ and increased its cooperativity by narrowing the phase transition peak. The most profound effects were seen in the presence of $10 \%$ Taxol, where $\mathrm{T}_{\mathrm{m}}$ increased and a second peak disappeared. On the other hand, the presence of $16 \%$ Taxol again lowered $\mathrm{T}_{\mathrm{m}}$, thus leading to re-appearance of the fluid phase. When added at $20 \%$, the drug affected only the glass transition temprature. Thus, in higher amounts, Taxol fluidized the phospholipid dispersion. In these circumstances, the glass transition temperature was more informative than the main phase transition temperature. These results were due to the chemical nature of the employed egg phosphatiylcholine and from the spatial properties of Taxol. Being a bulky molecule, the drug could not reach the inner parts of phospholipid hydrocarbon chains. It had only a limited access to hydrophobic parts and apparently bound to their surface acyl chans. The validity of this deduction remains to be tested further by employing for instance Electron Spin Resonance spectroscopy.

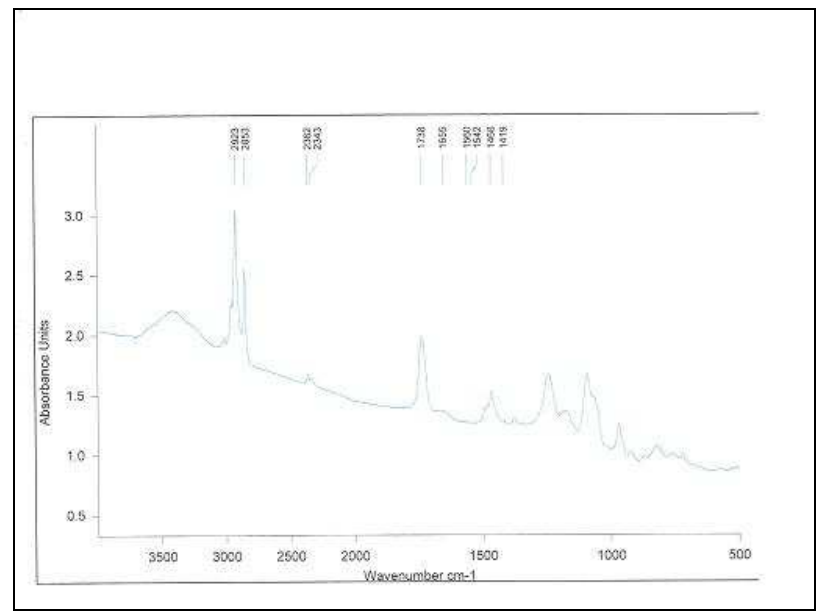

Figure 2 - Overal infrared spectrum of egg phosphatidylcholine. 


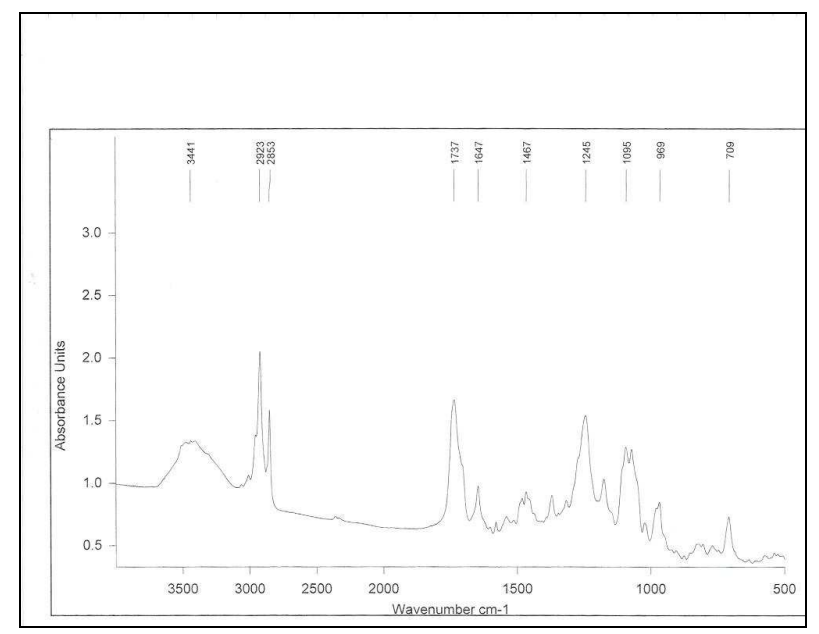

Figure 3 - The infrared spectrum of equimolar mixture of Taxol with egg phosphatidylcholine.

Having considered the advantages of vibrational spectroscopy over the other analytical methods for the analysis of drug-membrane phospholipid interactions (Günzler and Gremlich, 2002), the Fourier Transform Infrared Spectroscopy (FTIR) was applied for further characterization of molecular recognitions between Taxol and phosphatidylcholine. The most common method of sample preparation for Fourier Transform Infrared Spectroscopy is solid state analysis of molecues under study after their compression and mixing with $\mathrm{KBr}$. In their study, Meyer et al. (2004) showed that the compaction of the $\mathrm{KBr} / \mathrm{sample}$ mixtures did not foster the aggregation and remained conformationally unaltered by this process. The method is invaluable in determining the role of different functional groups engaged in such complexations (Seydel and Wiese, 2002). Fig. 2 and Fig. 3. show the overal infrared (IR) spectra of unbound drug and its equimolar mixure with the phospholipid, respectively. Since, using the excess quantities of Taxol created a drug dominated IR spectra of the binary mixtures (data not shown for brevity), only 1:1 mixture has been presented here, because it gave better impression of the recognition effects.

Under these conditions, the amorphous Taxol was converted to anhydrous state, suggesting that several solid-state structures of the drug coexisted. Major lipid specific band frequencies were seen when equimolar ratios of drug-lipid complexes were used. The most important of these were those belonging to $\mathrm{CH}$ vibrations $\left(2750-3100 \mathrm{~cm}^{-1}\right)$ and $\mathrm{CH}_{2}$ wagging progression and the $\mathrm{CH}_{2}$ scissoring vibrations (1150-1400 $\mathrm{cm}^{-1}$ and 1465-1475 $\left.\mathrm{cm}^{-1}\right)$.
These depicted the conformational order and provided information on packing effects of the acyl chains, respectively. $\mathrm{PO}_{2}^{-}$vibrations were located at $1242 \mathrm{~cm}^{-1}$. On the other hand, Taxol possessed substantial bands at 1600-1750, 11801300 and $630-770 \mathrm{~cm}^{-1}$. These were seen in the spectra of various drug-lipid mixtures, suggesting a drug dominated spectrum, indicating their recognition and binding. In comparison with the spectra of the unbound drug the lipid-bound spectral shits of Taxol's carbonyls from $1736 \mathrm{~cm}^{-1}$ to $1732 \mathrm{~cm}^{-1}$ and from $1736 \mathrm{~cm}^{-1}$ to $1707 \mathrm{~cm}^{-1}$ were seen (Fig. 3 and Table-1). These were retained also in higher drug ratios. The strong bands at $3300-3500 \mathrm{~cm}^{-1}$ were due to $\mathrm{OH}$-groups linked to the H-bonds. Following the drug-lipid recognition, the intensities were decreased and additional band shifts took place. This indicated a conformational restriction of lipid moiety due to bond formation with the drug, depicting the decrease in the drug flexibility. In conclusion, FTIR spectroscopy clearly showed that Taxol-lipid recognition and binding was governed by $\mathrm{CH}$ and $\mathrm{CH}_{2}$ groups, as well as $\mathrm{H}$-bonded surface $\mathrm{OH}$ groups. In addition, $\mathrm{PO}_{2}{ }^{-}, \mathrm{C}=\mathrm{O}, \mathrm{C}=\mathrm{C}$ and $\mathrm{CH}_{2}$ groups of the drug were also engaged. Data showed that Taxol fluidized the upper region of the acyl chains, whereas the hydrophobic core was rigidized. The increase in the asymmetric and symmetric methylene stretching frequencies, the splitting of methylene scissoring band and broadening of carbonyl stretching mode depicted that the drug mostly recognized the cooperativity region, bound to the surface phospholipid acyl chains and induced fluidity in their headgroup 
region. At higher amounts $(\geq 10 \%)$ it located at the interface and eventually formed a phase separation, resulting in crystallization, as deduced also from DSC measurements (Fig. 1).

Thus, by employing such a calorimetric and spectroscopic approaches, further details of Taxolmembrane phospholipid interactions could be obtained and exploited for elucidating its chemotherapeutic effects. Therefore, studying its recognition events with cellular phospholipids becomes crucial. Such biophysical and structural studies of Taxol-lipid arrangements can give valuable information about the organization of the drug in the membranes of both normal and malignant cells and can help to optimize the lipid matrix concerning its solubility potential. Moreover, the question of how higher amounts of the drug give rise to membrane deformation could be clarified in more detail. In this respect, a novel hypothesis can be generated regarding the fact that concentration dependent Taxol-induced membrane deformation may require physical perturbation of the lipid bilayer, a process in which the role of lipophilic drugs that partially penetrate the lipid bilayer is to be emphasized.

Table 1 - Principal infrared absorption bands, relative intensities and frequency assignments $\left(\mathrm{cm}^{-1}\right)$ for free lipid and its binary complex with Taxol.

\begin{tabular}{|c|c|c|}
\hline Phosphatidylcholine & Vibrational frequencies & Corresponding moieties \\
\hline & 3420 & water band \\
\hline & 2923 & $\mathrm{CH}$ stretching \\
\hline & 2853 & $\mathrm{CH}$ stretching \\
\hline & 2382 & $v_{s}\left[\left(\mathrm{CH}_{3}\right)_{4}\right]$ \\
\hline & 2342 & $v_{s}\left[\left(\mathrm{CH}_{3}\right)_{4}\right]$ \\
\hline & 1738 & $v_{s}[\mathrm{C}=\mathrm{O}]$ \\
\hline & 1556 & $\delta[\mathrm{C}-\mathrm{H}]$ \\
\hline & 1550 & $v_{a s}\left[\mathrm{PO}_{2}^{-}\right]$ \\
\hline & 1419 & scissoring $\delta\left[\left(\mathrm{CH}_{2}\right)_{\mathrm{n}}\right]$ \\
\hline \multicolumn{3}{|c|}{ Phosphatidylcholine- Taxol complex } \\
\hline & 709 & Finger print \\
\hline & 969 & Assymetric $\left[{ }^{+} \mathrm{N}-\left(\mathrm{CH}_{3}\right)_{3}\right]$ stretching \\
\hline & 1095 & Symmetric $v_{\mathrm{s}}\left[\mathrm{PO}_{2}^{-}\right]$ \\
\hline & 1245 & Antisymmetric $v_{\text {as }}\left[\mathrm{PO}_{2}^{-}\right]$ \\
\hline & 1467 & Scissoring $\mathrm{CH}_{2 \delta}$ (hexagonal) \\
\hline & 1647 & $\mathrm{C}=\mathrm{O} ;$ symmetric alyl $\mathrm{C}=\mathrm{C}$ \\
\hline & 1737 & $\mathrm{C}=\mathrm{O}$ stretching \\
\hline & 2853 & Symmetric $v_{\mathrm{s}}\left[\left(\mathrm{CH}_{3}\right)_{4}\right]$ \\
\hline & 2923 & Symmetric $v_{\mathrm{s}}\left[\left(\mathrm{CH}_{3}\right)_{4}\right]$ \\
\hline & 3441 & $\mathrm{H}$-bound $\mathrm{OH}$ groups \\
\hline
\end{tabular}

\section{ACKNOWLEDGEMENTS}

This work was supported by Gazi University Research Foundation (Project No: 02-2005/15). The author greatly acknowledges the facilities and personnel of The Central Laboratory of Middle East Technical University (Ankara-Turkey) for access to instruments.

\section{REFERENCES}

Castor, T. P. (2005), Phospholipid nanosomes. Curr Drug Deliv., 2, 4, 329-340.

H. Günzler and H.-U. Gremlich. (2002), IR Spectroscopy. An Introduction, Wiley-VCH Verlag GmbH, 69469, Weinheim, Germany. 
Harlan, Jr. W. R. (2001), New opportunities and proven approaches in complementary and altenative medicine research at the National Institutes of Health. J. Altern. Compl. Med., 7, S53.

Seydel, J. K. and Wiese, M. (2002), Drug-Membrane Interactions. Analysis, Drug Distribution, Modeling. In: R. Mannhold, H. Kubinyi and G. Folkers (Eds.). Methods and Principles in Medicinal Chemistry, Vol. 15, Wiley-VCH Verlag GmbH, Weinheim, Germany.

Hennenfent, K. L. and Govindan, R. (2006). Novel formulations of taxanes: a review. Old wine in a new bottle? Ann Oncol., 17, 735-749.

Meyer, J. D.; Manning, M. C. and Carpenter, J. F. (2004), Effects of potassium bromide disk formation on the infrared spectra of dried model proteins. $J$. Pharm. Sci, 93(2), 496-506.

Nornoo, A. O.; Osborne, D. W. and Chow, D. S. (2008) Cremophor-free intravenous microemulsions for paclitaxel I: formulation, cytotoxicity and hemolysis. Int. J. Pharm, 12; 349 (1-2), 108-16.

Nuijen, B., Bouma, M.; Schellens, J. H and Beijnen, J. H. (2001), Progress in the development of alternative pharmaceutical formulations of taxanes. Invest New Drugs, 19(2),143-153.

Safavy, A. (2008), Recent developments in taxane drug delivery. Curr. Drug. Deliv, 5 (1), 42-54.

Singla, A. K.; Garg, A. and Aggarwal, D. (2002), Paclitaxel and its formulations. Int. J. Pharm, 20, 235 (1-2), 179-192.
Stohs, S. J. (2005), Taxol in Cancer Treatment and Chemoprevention. In: Phytopharmaceuticals in Cancer Chemoprevention, Debasis Bagchi and Harry G. Preuss (Eds.), CRC Pres, Boca Raton, London, New York, Washington D. C., pp. 519-524.

Sznitowska, M.; Lunder, M. and Placzek, M. (2008), Paclitaxel solubility in aqueous dispersions and mixed micellar solutions. Chem. Pharm. Bull. 56 (1), 70-74.

Terwogt, J. M.; Nuijen, B.; Huinink, W. W. and Beijnen, J. H. (1997), Alternative formulations of paclitaxel. Cancer Treat. Rev, 23 (2), 87-95.

Wall, M. E. and Wani, M.C. (1995), Camptothecin and taxol: discovery to clinic (13th Bruce F. Caine Memorial Award Lecture), Cancer Res., 55, 753.

Wall, M. E. and Wani, M. C. (1995), Camptothecin and taxol: from discovery to clinic. $J$. Ethopharmacol., 51, 239.

Zefirova, O.; Nurieva, E.; Ryzhov, A.; Zyk, N. And Zefirov, N. (2005), Taxol: Synthesis, Bioactive Conformations, and Structure-Activity Relationships in Its Analogs; Russ. J. Org. Chem., 41, (3), 315-351 (37)

Received: February 06, 2009; Revised: October 19, 2009; Accepted: June 27, 2010. 